\title{
A systematic review of dietary assessments of pregnant adolescents in industrialised countries
}

\author{
Victoria Hall Moran* \\ Maternal and Infant Nutrition and Nurture Unit (MAINN), Brook Building, Faculty of Health, \\ University of Central Lancashire, Preston PRI 2HE, UK
}

(Received 5 April 2006 - Revised 26 August 2006 - Accepted 24 October 2006)

\begin{abstract}
Adolescent pregnancy is a major public health challenge for many industrialised countries and is associated with significant medical, nutritional, social and economic risk for mothers and their infants. Despite this, relatively little is known about the nutrient intakes of adolescents during pregnancy. The aim of this study is to review the current evidence relating to the dietary assessment of pregnant adolescents living in industrialised countries. Nine papers were identified that fulfilled the inclusion criteria, seven of which were conducted in the USA. Mean nutrient intakes were compared with the most recent US dietary reference intakes (DRI) for pregnant adolescents. Despite the poor quality of the majority of studies, there appeared to be some consensus to suggest that the nutrient intakes of pregnant adolescents were below the DRI for energy, iron, folate, calcium, vitamin E and magnesium, nutrients which are recognised to be vital for fetal growth and development during pregnancy. Modest differences were observed in nutrient intake between trimesters and age groups. Current research is limited by sampling and measurement bias, and research is urgently required to address these limitations. Further consideration should also be made of the influence of age and of role of socio-economic support on pregnant adolescents' nutrient intake. The achievement of improved nutrition in pregnancy among adolescents requires multidisciplinary collaborations of adolescent health care providers, academics, professional organisations, policy makers, industry and service users. Only once this is achieved can adolescent nutrition, and adolescent nutrition in pregnancy, be significantly and sustainably optimised.
\end{abstract}

Dietary assessment: Pregnancy: Adolescent: Systematic review

'In the world's rich nations, more than three quarters of a million teenagers will become mothers in the next twelve months'

(UNICEF, 2001, p. 3)

Adolescent pregnancy is a major public health challenge for many industrialised countries. In the most up-to-date and comprehensive survey of adolescent birth rates in the industrialised world, it was reported in 1998 that the USA had the highest adolescent birth rate, whilst the UK had the highest adolescent birth rate in Europe. Rates of adolescent births (the number of births per 1000 adolescents aged 15-19 years) in the USA were $52 \cdot 1$ per 1000 births - about four times the European Union average. Adolescent birth rates in the UK were 30.8 per 1000 births - approximately five times those of The Netherlands, three times those of France and twice those of Germany (UNICEF, 2001). Although there is some evidence to suggest that these rates have fallen in recent years, for example the under 18 and under 16 conception rates in England have decreased by 9.8 and 9.9\% respectively since 1998 (Office for National Statistics, 2005), adolescent pregnancy rates remain high.

Adolescent pregnancy is associated with significant medical, nutritional, social and economic risk for mothers and their infants. There is evidence to suggest that the medical risk is particularly severe for young adolescents. Infants born to young adolescents $(<15$ years $)$ are twice as likely to be low birth weight $(\mathrm{LBW})(<2500 \mathrm{~g})$ and three times as likely not to survive the neonatal period compared with infants born to adult mothers (Lenders et al. 2000). Recent UK data confirm that children born to adolescent mothers have the highest infant mortality rate of 7.9 per 1000 live births. This contrasts with a rate of 4.3 per 1000 live births in women aged 30-34 years (the lowest risk group) (Office for National Statistics, 2004). Rates of spontaneous miscarriage and of very preterm birth $(<32$ weeks) are highest in those aged 13-15 years (Olausson et al. 1999). Young adolescent mothers are at higher risk for maternal complications than adult mothers, e.g. abnormally high maternal weight gains, pregnancyinduced hypertension, pre-eclampsia, anaemia and renal disease (Hediger et al. 1990; Story \& Alton, 1995; Lenders et al. 2000; Umans \& Lindheimer, 2001).

The cause of adverse pregnancy outcome in the adolescent has been debated. Some attribute the poor outcomes to various factors associated with being young, e.g. poor socio-economic status, lifestyle and adequacy of prenatal care, factors which are risk factors for poor birth outcomes in their own right.

Abbreviations: AI, adequate intake; DRI, dietary reference intakes; EAR, estimated average requirements; EER, estimated energy requirement; LBW, low birth weight.

* Corresponding author: Dr V. Hall Moran, fax +44 1772 892914, email vlmoran@uclan.ac.uk 
For example, it has been reported that, in the UK, adolescent mothers are more likely than older mothers to come from unskilled manual backgrounds or live in areas with higher social deprivation; have mother's who were adolescent mothers themselves; have low self-esteem; have low educational achievement (Teenage Pregnancy Unit, 2004); and are more likely than older mothers to smoke during pregnancy (Hamlyn et al. 2002). Pregnant adolescents are also more likely to enter prenatal care late and less likely to obtain an adequate quantity of care compared with adults (StevensSimon et al. 1992). Casanueva and colleagues (1991) investigated the effect of late prenatal care on the nutritional status of 163 pregnant adolescents aged 11-17 years (mean age 15 years (SD 1)) and found that late prenatal care (accessed when $\geq 25$ weeks pregnant) was associated with increased risk of maternal anaemia, iron deficiency and zinc deficiency.

Other researchers have attributed the poor pregnancy outcomes of adolescent mothers to an independent factor related to some aspect of the woman's physiology, such as gynaecological immaturity, competition for nutrients, or the growth and nutritional status of the mother (King, 2003). A plausible explanation for the negative effect of young gynaecological age on pregnancy outcome is the competition for nutrients between the mother and fetus. The competition for nutrients hypothesis was first proposed by Naeye (1981). Further support for the hypothesis was provided by a study that reported that infants born to young, growing mothers were smaller than those born to adult women (Frisancho et al. 1983). In more recent research, Scholl et al. $(1990,1994)$ reported that many pregnant adolescents continue to grow during gestation, as assessed by measuring knee height length, and that these adolescents give birth to smaller infants (about $155 \mathrm{~g}$ less) despite a tendency to gain more weight during pregnancy and retain more weight postpartum than non-growing adolescents. In their subsequent research, Scholl et al. (2000) found that growing adolescents have a surge in maternal leptin concentrations during the last trimester, which may reduce the rate of maternal fat breakdown during late pregnancy and thereby increase the mother's use of glucose for energy. This would result in less energy being available for fetal growth. Therefore, it looks possible that the still-growing pregnant adolescent partitions metabolic fuels to enable more energy to become available for maternal growth (and therefore higher maternal fat gains) at the expense of that available for fetal growth (resulting in lower birth weights).

Adolescence is a critical period during which lifetime habits are established (Cavadini et al. 2000) and, as adolescents are particularly susceptible to certain risk behaviours, including unhealthy eating, the impact that their eating behaviour has on both their short- and long-term nutritional status is considerable. Nutritional surveys have shown that the highest prevalence of nutritional deficiencies occurs in adolescence, with the most commonly noted deficiencies in calcium, iron, zinc, riboflavin, folate and vitamins A and D (e.g. Gregory et al. 2000). The dietary habits acquired during adolescence have the potential to enhance or undermine health throughout life. For example, high fat intake during adolescence and into adulthood is associated with an increased risk of heart disease, and low calcium intake during adolescence is associated with low bone density and an increased risk of osteoporosis in later life (Lytle, 2002). Adequate nutrition in adolescent pregnancy therefore presents many challenges for health professionals and policy makers alike. Despite this, relatively little is known about the nutrient intakes of adolescents during pregnancy. The aim of this study is to review the current evidence relating to the dietary assessment of pregnant adolescents living in industrialised countries.

'Adolescence' can be described as the transitional stage of development between childhood and adulthood. It is a cultural and social phenomenon and therefore its end points are not easily defined. Stedman's Medical Dictionary defines an adolescent as: 'a young person who has undergone puberty but who has not reached full maturity; a teenager' (2004). For reasons of clarity, here 'adolescence' refers to the ages 13-19 years inclusive (unless otherwise stated).

\section{Methods}

The research question applied to the systematic review was 'what is the nature of the nutrient intakes of pregnant adolescents living in industrialised countries?' Papers that focused on adolescents living in non-industrialised countries were not included in this review due to the cultural and socio-economic disparities between the industrialised and non-industrialised world, making synthesis of findings impractical. The systematic review protocol broadly followed the NHS Centre for Reviews and Dissemination guidelines (2001). The main stages of the review are illustrated in Fig. 1.

Stage 1 of the review involved searching for publications using electronic databases, websites and citations, hand searching relevant journals and contacts with experts. The search terms used in the electronic databases were: pregnant, pregnancy, maternal, mother, nutrition, nutrient, food, adolescent, adolescence, teenager, teenage, teen, young, child, girl. The databases searched were: Ovid Medline, CINAHL, British Nursing Index, Proquest Nursing Journals, The Cochrane Library and EBSCO Host Electronic Journals Service. The Journal of the American Dietetic Association and the British Journal of Nutrition were hand searched. Studies published between 1980 and 2006 were included in the review.

At stage 2 of the review, the title and abstracts were analysed for relevance to the research question and the inclusion and exclusion criteria were applied (see Table 1). Primary papers were then obtained and further scrutinised against the inclusion and exclusion criteria (stage 3). Studies that fulfilled the inclusion criteria were then assessed for quality (stage 4) using quality criteria for quantitative studies (CASP, 2004).

\section{Analysis}

Given the methodological variation in the included papers, the papers were tabulated and summarised narratively. Mean nutrient intake data were compared with the most recent US dietary reference intakes (DRI) for pregnant adolescents, as published by the Institute of Medicine, Food and Nutrition Board (1997, 1998, 2000a,b, 2005). US recommendations were used for purposes of clarity and consistency of comparison, as all but one of the studies was conducted in the USA or US territories. When available, mean nutrient values were compared with the estimated average requirements (EAR). The EAR is defined as 'the average daily nutrient intake level estimated to meet the requirement of half the healthy 


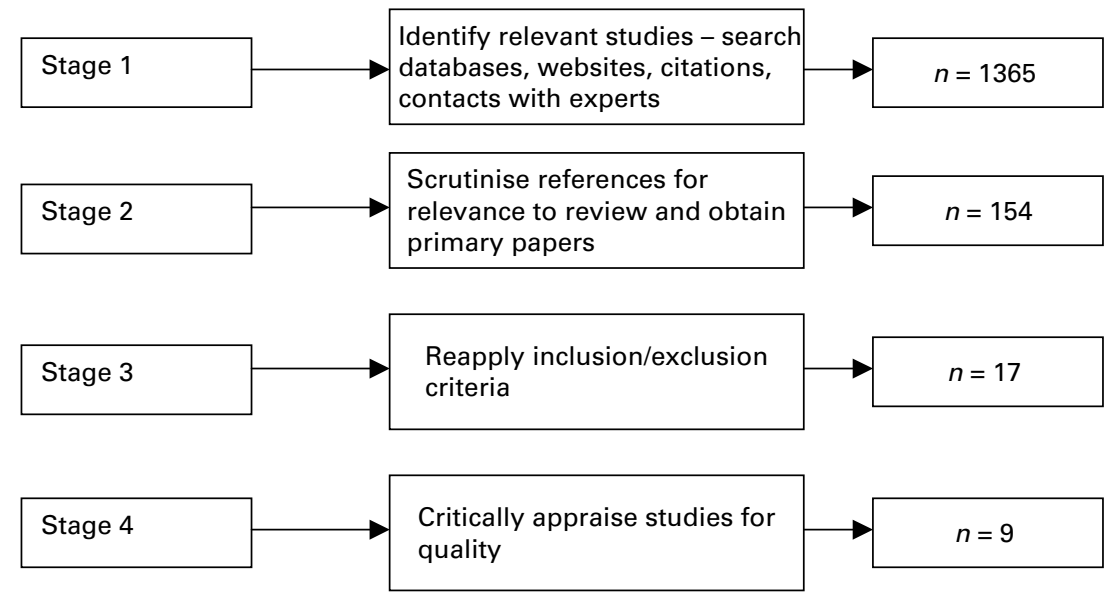

Fig. 1. Main stages of the systematic review process.

individuals in a particular life stage and gender group' (Institute of Medicine, Food \& Nutrition Board, 2005, p. 3). Intakes were compared with the EAR in preference to the RDA because, even when the mean nutrient intake of a group is found to equal the RDA, a proportion of persons in the group will still have usual intakes below the EAR due to the large between-subject variation in nutrient intakes. To ensure a low prevalence of intakes below the EAR, the mean intake of a group should exceed the RDA, often by a considerable amount (Gibson, personal communication, 2005). Ideally, the proportion of adolescents meeting or failing to meet the reference standard would be provided in order to evaluate the adequacy of nutrient intakes of a population group. As information on the number of adolescents whose intake falls below the reference standard is unavailable in the majority of studies, the group mean will be compared with the reference standard. For nutrients that do not yet have a defined EAR, the AI (adequate intakes) or RDA were used as reference values. Energy intakes were compared with the estimated energy requirement (EER). In pregnant and lactating women, the EER incorporates 'the needs associated with the deposition of tissues or the secretion of milk at

Table 1. Inclusion and exclusion criteria

\begin{tabular}{|c|c|c|}
\hline Aspect & Inclusion criteria & Exclusion criteria \\
\hline Types of study & Research papers using any methods & $\begin{array}{l}\text { Opinion papers } \\
\text { Letters to editor } \\
\text { Foreign language }\end{array}$ \\
\hline Focus of study & $\begin{array}{l}\text { Studies designed to assess the } \\
\text { nutrient intakes of pregnant adolescents } \\
\text { Studies with emergent findings relating } \\
\text { to nutrient intakes of pregnant } \\
\text { adolescents }\end{array}$ & \\
\hline Participants & $\begin{array}{l}\text { Healthy pregnant human adolescents living } \\
\text { in industrialised countries }\end{array}$ & $\begin{array}{l}\text { Non-human studies } \\
\text { Pregnant adults } \\
\text { Non-pregnant or unwell adolescents } \\
\text { Adolescents living in non-industrialised countries }\end{array}$ \\
\hline Presentation and analysis of data & $\begin{array}{l}\text { Papers presenting nutrient intakes as } \\
\text { absolute values }\end{array}$ & 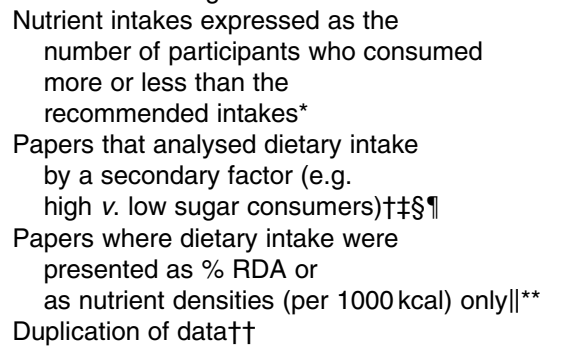 \\
\hline
\end{tabular}

\footnotetext{
* Burchett \& Seely (2003).

† Lenders et al. (1994).

‡Lenders et al. (1997).

$\S$ Chang et al. (2003).

I Carruth (1981).

\| Endres et al. (1987)

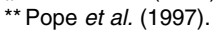

†† Skinner \& Carruth (1991).
} 
rates consistent with good health' (Institute of Medicine, Food \& Nutrition Board, 2005, p 3).

\section{Findings}

Nine papers were identified that fulfilled the inclusion criteria (see Table 2). Seven of the papers were conducted in the USA, one was conducted in a US territory (Guam, an island located in the western North Pacific Ocean) and one was carried out in Australia. In all but three of the papers, the nutrient intake of adolescents was the primary outcome of interest. Gutierrez's (1999) main outcome measures were maternal weight gain and infant birth weight, whereas Job \& Capra (1995) and Giddens et al. (2000) conducted a comparative evaluation of the nutrient intakes of pregnant adolescents and pregnant adults. Job \& Capra (1995) also compared the intakes of pregnant adolescents and non-pregnant adolescents. Three papers conducted secondary statistical analyses on nutrient intakes at different stages of pregnancy (Job \& Capra, 1995; Giddens et al. 2000; Pobocik et al. 2003), whilst another compared the intake in two trimesters descriptively (Gutierrez, 1999). One paper compared nutrient intakes of pregnant adolescents before and during participation in a supplemental food programme (Endres et al. 1985) and two sought to determine whether differences were present among various age groups of adolescents (Job \& Capra, 1995; Pobocik et al. 2003).

\section{Participants}

Participants were aged 13-20 years, with a mean age of between 16 and 17 years. Only one study randomly selected their participants (Giddens et al. 2000); the rest used convenience samples. Eligibility criteria for participation were often limited, with only two studies employing exclusion criteria. Gutierrez (1999) excluded multigravida adolescents and those with a history of miscarriage or health problems (e.g. diabetes, kidney or heart problems) and competitive athletes or heavy exercisers, and Job \& Capra (1995) excluded adolescents with gestational diabetes. Few of the studies provided clear or detailed information regarding the socio-economic status of the participants in their study. In some studies socio-economic status was implied, whether it was a mix of low and high socio-economic groups (Loris et al. 1985) or predominantly a low socio-economic group (Carruth \& Skinner, 1991; Giddens et al. 2000; Pobocik et al. 2003). Two studies provided more detailed information, indicating that their sample consisted of low socio-economic group participants (Endres et al. 1985, Skinner et al. 1992). Two of the studies related to very specific populations, i.e. Mexican American (Gutierrez, 1999) and Guamananian adolescents (Pobocik et al. 2003), both of whom are subject to specific cultural influences limiting the generalisability of these studies to different populations.

\section{Data collection}

Data collection methods varied widely between studies, ranging from single $24 \mathrm{~h}$ recalls to $7 \mathrm{~d}$ estimated food records, as described in Table 2. The measures utilised to ensure the validity of the data collection methods were diverse and often inadequate. Only three studies specified whether the data collector was trained and/or appropriately qualified (Endres et al. 1985; Skinner et al. 1992; Giddens et al. 2000), two trained their participants in the data collection method used (Skinner et al. 1992; Giddens et al. 2000) and five used models, photographs and/or household measures to aid the estimation of portion sizes (Loris et al. 1985; Skinner et al. 1992; Job \& Capra, 1995; Giddens et al. 2000; Pobocik et al. 2003). Although some did not specify clearly, it appeared that no studies included the contribution of supplemental vitamins or minerals in their nutrient calculations. Five studies conducted both weekday and weekend assessment of dietary intake (Carruth \& Skinner, 1991; Skinner et al. 1992; Job \& Capra, 1995; Gutierrez, 1999; Giddens et al. 2000) and two carried out measurements during different seasons (Job \& Capra, 1995; Giddens et al. 2000). Only one study collected data regarding the nutritional status of pregnant adolescents. Endres et al. (1985) reported $\mathrm{Hb}$ and haematocrit values, but provided no methodological detail about the measurements. Pregnancy outcomes were described in three studies: namely infant birth weight (Loris et al. 1985; Gutierrez, 1999) and maternal weight gain (Loris et al. 1985; Gutierrez, 1999; Giddens et al. 2000). Mean infant birth weight ranged from $3288 \mathrm{~g}$ (Gutierrez, 1999) to $3377 \mathrm{~g}$ (Loris et al. 1985), comparing well with reported norms. The WHO Collaborative Study on Maternal Anthropometry and Pregnancy Outcomes showed that birth weights between 3.1 and $3.6 \mathrm{~kg}$, with a mean of $3.3 \mathrm{~kg}$, were associated with the optimal ratio of good fetal and maternal outcomes (WHO, 1995; Kelly et al. 1996). Gutierrez (1999) reported that 45 of the 46 births resulted in healthy term infants, whereas eight of 135 infants in Loris and colleagues' study were born preterm and five were small for gestational age $(<2500 \mathrm{~g})$. Mean total maternal weight gains were $14.2 \mathrm{~kg}$ (Giddens et al. 2000), $14.4 \mathrm{~kg}$ (Gutierrez, 1999) and $16.8 \mathrm{~kg}$ (Loris et al. 1985). These average weight gains were somewhat higher than recent recommendations that healthy, well-nourished women should gain 10-14 kg during pregnancy, with an average of $12 \mathrm{~kg}$, in order to increase the probability of delivering full-term infants with an average birth weight of $3.3 \mathrm{~kg}$, and to reduce the risk of fetal and maternal complications (FAO/WHO/UNU, 2004). All studies, however, were limited by the lack of knowledge of pre-pregnancy BMI, relying instead on use of weight at the first prenatal visit or maternal recollection.

\section{Nutrient intake}

Mean nutrient intake data were compared with current US DRI. The nutrient intakes that fell most frequently below the DRI were energy, iron, folate, calcium, vitamin $\mathrm{E}$ and magnesium (Table 3).

All nine studies reported that iron intakes of pregnant adolescents fell below the EAR of $23 \mathrm{mg} / \mathrm{d}$. Mean iron intakes were found to be lower in the second than the third trimester of pregnancy $(P<0 \cdot 05$, Pobocik et al. 2003), although Job \& Capra (1995) and Giddens et al. (2000) failed to find any statistical difference. Eight studies found that energy intakes were below the EER. Gutierrez (1999) reported that adolescents tended to consume approximately 300 calories more during the third trimester compared with the second trimester, but no statistically significant differences were observed. All of 
Table 2. Characteristics of included studies

\begin{tabular}{|c|c|c|c|c|c|c|}
\hline \multirow[b]{2}{*}{ Author and country } & \multicolumn{2}{|c|}{ Participants } & \multirow{2}{*}{$\begin{array}{l}\text { Methods } \\
\text { Data collection }\end{array}$} & \multicolumn{2}{|l|}{ Results } & \multirow[b]{2}{*}{ Quality } \\
\hline & Age/number & Inclusion criteria & & Participants & Dietary intake & \\
\hline \multirow[t]{2}{*}{$\begin{array}{l}\text { Endres et al. (1985) } \\
\qquad \text { USA }\end{array}$} & $\begin{array}{l}\text { Forty-six WIC } \\
\text { pregnant adolescents } \\
\text { and ninety-one } \\
\text { pre-WIC pregnant } \\
\text { adolescents aged } \\
15-18 \text { years }\end{array}$ & $\begin{array}{l}\text { All participants } \\
\text { met the criteria } \\
\text { for the WIC } \\
\text { programme } \\
\text { ( } 185 \% \text { of poverty) }\end{array}$ & $\begin{array}{l}24 \mathrm{~h} \text { recall Energy } \\
\text { and thirteen } \\
\text { nutrients assessed }\end{array}$ & $\begin{array}{l}\text { WIC adolescents had received } \\
\text { supplemental food for average } \\
\text { of } 4 \text { months }\end{array}$ & $\begin{array}{l}\text { Nutrient intakes } \\
\text { below US DRI: } \\
\text { energy, calcium, } \\
\text { iron, folate, } \\
\text { vitamin } \mathrm{E} \text {, vitamin } \mathrm{B}_{6}\end{array}$ & $\begin{array}{l}\text { Data collection: data collected by } \\
\text { trained interviewers; no details } \\
\text { of day of week data collected; } \\
\text { unclear whether data included } \\
\text { nutrient intake from supplements }\end{array}$ \\
\hline & & No exclusions & $\begin{array}{l}\text { Data collected at } 26 \\
\text { weeks (WIC) and } \\
20 \text { weeks } \\
\text { (pre-WIC) (means) }\end{array}$ & & & $\begin{array}{l}\text { Participants: mean age not given; } \\
\text { convenience sample; low } \\
\text { socio-economic group; no } \\
\text { exclusion criteria utilised }\end{array}$ \\
\hline \multirow[t]{2}{*}{$\begin{array}{l}\text { Loris et al. (1985) } \\
\qquad \text { USA }\end{array}$} & $\begin{array}{l}\text { Fifty-seven } \\
\text { pregnant } \\
\text { adolescents aged } \\
13-19 \text { years }\end{array}$ & $\begin{array}{l}\text { Singleton } \\
\text { births, recruited } \\
\text { from two areas: } \\
\text { one predominantly } \\
\text { low-income, one } \\
\text { middle-/upper-income }\end{array}$ & $\begin{array}{l}24 \mathrm{~h} \text { recall and FFQ } \\
\text { Energy and ten } \\
\quad \text { nutrients assessed }\end{array}$ & $\begin{array}{l}27 \% \text { of participants received } \\
\text { food stamps; } 23 \% \text { enrolled } \\
\text { in WIC }\end{array}$ & $\begin{array}{l}\text { Nutrient intakes below } \\
\text { US DRI: iron }\end{array}$ & $\begin{array}{l}\text { Data collection: portions estimated } \\
\text { using household measures; no } \\
\text { training provided; no details of } \\
\text { day of week data collected; } \\
\text { unclear whether data included } \\
\text { nutrient intake from supplements }\end{array}$ \\
\hline & & No exclusions & $\begin{array}{l}\text { Data collected in } \\
\text { second or third } \\
\text { trimester }\end{array}$ & & & $\begin{array}{l}\text { Participants: mean age not given; } \\
\text { convenience sample; range of } \\
\text { socio-economic groups suggested; } \\
\text { limited exclusion criteria utilised }\end{array}$ \\
\hline \multirow[t]{2}{*}{$\begin{array}{l}\text { Carruth \& Skinner } \\
\text { (1991) USA }\end{array}$} & $\begin{array}{l}\text { Thirty-four } \\
\text { pregnant } \\
\text { adolescents } \\
\text { aged 13-18 } \\
\text { years }\end{array}$ & No exclusions & $\begin{array}{l}24 \mathrm{~h} \text { recall and dietary } \\
\text { records of two } \\
\text { weekdays and } \\
\text { one weekend day }\end{array}$ & $\begin{array}{l}\text { Mean age at conception } 16.6 \text { years; } \\
\text { mean gynaecological age } \\
4.0+1.9 \text { years. }\end{array}$ & $\begin{array}{l}\text { Nutrient intakes below } \\
\text { US DRI: energy, } \\
\text { calcium, iron }\end{array}$ & $\begin{array}{l}\text { Data collection: no details of measures } \\
\text { used to ensure validity of data } \\
\text { collection tools (no models used, no } \\
\text { training, etc.); both weekday and } \\
\text { weekend days assessed; nutrient } \\
\text { intake from food sources only }\end{array}$ \\
\hline & & & $\begin{array}{l}\text { Energy and } \\
\text { nine nutrients } \\
\text { assessed }\end{array}$ & $\begin{array}{l}\text { Twenty-seven white, seven black; nine } \\
\text { lived with both parents, remainder } \\
\text { lived with single parents/husbands; } \\
\text { three-quarters single; twenty received } \\
\text { welfare, one-third of their families } \\
\text { received financial assistance }\end{array}$ & & $\begin{array}{l}\text { Participants: standard deviation of } \\
\text { age not given; convenience } \\
\text { sample; some detail of } \\
\text { socio-economic grouping; no } \\
\text { exclusion criteria utilised }\end{array}$ \\
\hline \multirow[t]{3}{*}{$\begin{array}{l}\text { Skinner et al. (1992) } \\
\text { USA }\end{array}$} & $\begin{array}{l}115 \text { pregnant adolescents } \\
\text { aged } 13-18 \text { years }\end{array}$ & $\begin{array}{l}\text { White pregnant } \\
\text { adolescents; } \\
<18 \text { years old } \\
\text { at conception }\end{array}$ & $\begin{array}{l}\text { Data collected } \\
\text { at } 28-36 \text { weeks } \\
\text { Two } 24 \mathrm{~h} \text { recalls } \\
\text { and } 2 \mathrm{~d} \text { dietary } \\
\text { records, including } \\
\text { one weekend day }\end{array}$ & $\begin{array}{l}\text { Mean age } 16 \cdot 3+1 \cdot 2 \text { years; } \\
\text { mean Hollingshead index score } \\
26 \cdot 4+9 \cdot 6 \text { (of a maximum of } 66 \text { ) }\end{array}$ & $\begin{array}{l}\text { Nutrient intakes } \\
\text { below US DRI: } \\
\text { energy. iron, folate, } \\
\text { magnesium }\end{array}$ & $\begin{array}{l}\text { Data collection: food models and } \\
\text { measuring utensils used, data } \\
\text { taken by registered dietitian, } \\
\text { training in food records given, } \\
\text { food records checked for } \\
\text { accuracy; both weekday and } \\
\text { weekend days assessed; } \\
\text { unclear if data included } \\
\text { nutrient intake from supplements }\end{array}$ \\
\hline & & No exclusions & $\begin{array}{l}\text { Energy and thirteen } \\
\text { nutrients assessed }\end{array}$ & & & $\begin{array}{l}\text { Participants: convenience sample; } \\
\text { Hollingshead score of } \\
\text { socio-economic status reported, } \\
\text { limited exclusion criteria utilised }\end{array}$ \\
\hline & & & $\begin{array}{l}\text { Data collected } \\
\text { in third trimester }\end{array}$ & & & \\
\hline
\end{tabular}




\begin{tabular}{|c|c|c|c|c|c|c|}
\hline \multirow[b]{2}{*}{ Author and country } & \multicolumn{2}{|c|}{ Participants } & \multirow{2}{*}{$\begin{array}{c}\text { Methods } \\
\text { Data collection }\end{array}$} & \multicolumn{2}{|l|}{ Results } & \multirow[b]{2}{*}{ Quality } \\
\hline & Age/number & Inclusion criteria & & Participants & Dietary intake & \\
\hline \multirow[t]{2}{*}{$\begin{array}{l}\text { Dunn et al. (1994) } \\
\text { USA }\end{array}$} & $\begin{array}{l}\text { Thirty } \\
\text { pregnant } \\
\text { 'teenagers' }\end{array}$ & $\begin{array}{l}\text { Participants } \\
\text { from a rural } \\
\text { southern } \\
\text { community }\end{array}$ & $\begin{array}{l}24 \mathrm{~h} \text { recall } \\
\text { Energy and } \\
\text { twenty-seven } \\
\text { nutrients } \\
\text { assessed }\end{array}$ & $\begin{array}{l}\text { Mean age } 16 \\
\text { years (three }<14 \text { years); } \\
\text { twenty-one black, eight } \\
\text { white, one Hispanic; mean } \\
\text { age at menarche } 11 \text { years; } \\
\text { mean gravida one; } \\
\text { mean parity zero; mean } \\
\text { gynaecological age } 6 \text { years }\end{array}$ & $\begin{array}{l}\text { Nutrient intakes } \\
\text { below US DRI: } \\
\text { energy, dietary } \\
\text { fibre, calcium, } \\
\text { iron, folate, } \\
\text { vitamin E, } \\
\text { magnesium, } \\
\text { potassium }\end{array}$ & $\begin{array}{l}\text { Data collection: no details of } \\
\text { measures used to ensure validity } \\
\text { of data collection tools (no models } \\
\text { used, no training, etc.); no details } \\
\text { of day of week data collected; } \\
\text { nutrient intake from food sources } \\
\text { only; no details of stage of } \\
\text { pregnancy }\end{array}$ \\
\hline & & No exclusions & $\begin{array}{l}\text { No details } \\
\text { of stage of } \\
\text { pregnancy } \\
\text { data given }\end{array}$ & & & $\begin{array}{l}\text { Participants: range and standard } \\
\text { deviation of age not given; } \\
\text { convenience sample; no } \\
\text { socio-economic data given; no } \\
\text { exclusion criteria }\end{array}$ \\
\hline \multirow[t]{3}{*}{$\begin{array}{l}\text { Job \& Capra (1995) } \\
\quad \text { Australia }\end{array}$} & $\begin{array}{l}\text { Thirty-five } \\
\text { pregnant } \\
\text { adolescents } \\
\text { aged } \\
15-18 \text { years }\end{array}$ & $\begin{array}{l}\text { Age } \leq 18 \text { years } \\
\text { Exclusions: } \\
\text { gestational } \\
\text { diabetes }\end{array}$ & $\begin{array}{l}\text { Between one } \\
\text { and three } \\
24 \mathrm{~h} \text { recalls. } \\
\text { FFQ used } \\
\text { to help } \\
\text { validate } \\
24 \mathrm{~h} \text { recall }\end{array}$ & $\begin{array}{l}\text { Mean age } \\
17 \text { years (range } 15-18 \text { years); } \\
\text { 'generally single and supported } \\
\text { by families'; 'majority' not completed } \\
\text { nor intended to complete tertiary } \\
\text { education; most Australian born }\end{array}$ & $\begin{array}{l}\text { Nutrient } \\
\text { intakes below } \\
\text { US DRI: energy, } \\
\text { calcium, iron, zinc }\end{array}$ & $\begin{array}{l}\text { Data collection: probing and life-size } \\
\text { colour photographs used to } \\
\text { estimate portion sizes; no } \\
\text { training given; interviews } \\
\text { sometimes by telephone; } \\
\text { interviews conducted on } \\
\text { different days of week and in } \\
\text { different seasons; FFQ used to } \\
\text { validate } 24 \mathrm{~h} \text { recall data; } \\
\text { nutrient intake from food } \\
\text { sources only; data collected in } \\
\text { three trimesters of pregnancy }\end{array}$ \\
\hline & & & & & $\begin{array}{l}\text { No significant } \\
\text { differences were } \\
\text { observed between } \\
\text { trimesters }\end{array}$ & $\begin{array}{l}\text { Participants: standard deviation } \\
\text { of age not given; convenience } \\
\text { sample; no socio-economic } \\
\text { data given; limited exclusion } \\
\text { criteria }\end{array}$ \\
\hline & & & $\begin{array}{l}\text { Energy and six } \\
\text { nutrients assessed } \\
\text { Data collected in first, } \\
\text { second and/or third } \\
\text { trimester }{ }^{\star}\end{array}$ & & & $\begin{array}{l}\text { Data analysis: statistical comparison } \\
\text { of stage of pregnancy }\end{array}$ \\
\hline $\begin{array}{l}\text { Gutierrez (1999) } \\
\text { USA }\end{array}$ & $\begin{array}{l}\text { Forty-six } \\
\text { pregnant } \\
\text { adolescents } \\
\text { aged } 13-18 \\
\text { years }\end{array}$ & $\begin{array}{l}\text { Inclusions: } \\
\text { primigradida; } \\
\text { Mexican } \\
\text { American; } \\
\text { aged 13-18 } \\
\text { years }\end{array}$ & $\begin{array}{l}\text { Two } 24 \mathrm{~h} \text { recalls } \\
\text { Energy and } \\
\quad \text { eleven nutrients } \\
\quad \text { assessed }\end{array}$ & $\begin{array}{l}\text { Mean age } 16 \cdot 34+1.56 \text { years } \\
\text { (13-18 years); ten US-born, } \\
\text { thirty-six Mexican-born; forty-five } \\
\text { healthy full-term infants and one } \\
\text { preterm; details of living arrangements, } \\
\text { income, employment status, } \\
\text { financial resources/services } \\
\text { accessed provided }\end{array}$ & $\begin{array}{l}\text { Nutrient intakes } \\
\text { below US DRI†: } \\
\text { energy, iron, } \\
\text { folate, vitamin E }\end{array}$ & $\begin{array}{l}\text { Data collection: no details of measures } \\
\text { used to ensure validity of data } \\
\text { collection tools (no models used, } \\
\text { no training, etc.); interviews } \\
\text { conducted by bilingual graduate } \\
\text { students; data collected on all days } \\
\text { of the week; data collected over } \\
\text { 18-month period; unclear whether } \\
\text { data included nutrient intake from } \\
\text { supplements; data collected } \\
\text { in two trimesters of pregnancy }\end{array}$ \\
\hline
\end{tabular}




\begin{tabular}{|c|c|c|c|c|c|c|}
\hline \multirow[b]{2}{*}{ Author and country } & \multicolumn{2}{|c|}{ Participants } & \multirow{2}{*}{$\begin{array}{c}\text { Methods } \\
\text { Data collection }\end{array}$} & \multicolumn{2}{|l|}{ Results } & \multirow[b]{2}{*}{ Quality } \\
\hline & Age/number & Inclusion criteria & & Participants & Dietary intake & \\
\hline & & $\begin{array}{l}\text { Exclusions: } \\
\text { history of miscarriage, } \\
\text { health problems } \\
\text { (e.g. diabetes, kidney } \\
\text { or heart problems), } \\
\text { competitive athletes, } \\
\text { heavy exercisers }\end{array}$ & $\begin{array}{l}\text { Data collected } \\
\text { in second } \\
\text { trimester } \\
\text { (18-22 weeks) } \\
\text { and third } \\
\text { trimester } \\
\text { (30-34 weeks) }\end{array}$ & & & $\begin{array}{l}\text { Participants: convenience sample; } \\
\text { socio-economic data provided; } \\
\text { exclusion criteria applied }\end{array}$ \\
\hline & & & & & & $\begin{array}{l}\text { Data analysis: descriptive comparison } \\
\text { of stage of pregnancy }\end{array}$ \\
\hline \multirow[t]{3}{*}{$\begin{array}{l}\text { Giddens et al. } \\
(2000) \text { USA }\end{array}$} & $\begin{array}{l}\text { Fifty-nine } \\
\text { pregnant } \\
\text { adolescents } \\
\text { aged 13-18 } \\
\text { years }\end{array}$ & $\begin{array}{l}\text { Inclusions: } \\
\text { all subjects } \\
\text { participating } \\
\text { in a larger } \\
\text { RCT on } \\
\text { calcium intake; } \\
\text { singleton } \\
\text { pregnancy; } \\
\text { any ethnicity; } \\
13-19 \text { weeks } \\
\text { gestation; all } \\
\text { prescribed } \\
\text { standard } \\
\text { multivitamin/mineral } \\
\text { prenatal supplement }\end{array}$ & Two $7 \mathrm{~d}$ food records & $\begin{array}{l}\text { Mean age } 16.9+1.3 \text { yeard (range } \\
14-18 \text { years); } 73 \% \text { African American, } \\
27 \% \text { white; } 10 \% \text { smokers; none } \\
\text { married; mean height } 161.6+3.9 \mathrm{~cm} ; \\
\text { approximately } 95 \% \text { of those attending } \\
\text { the prenatal recruitment clinics had } \\
\text { household income at or below } 185 \% \\
\text { poverty level }\end{array}$ & $\begin{array}{l}\text { Nutrient intakes } \\
\text { below US DRI: } \\
\text { energy, dietary } \\
\text { fibre, calcium, } \\
\text { iron, folate, } \\
\text { vitamin E, } \\
\text { magnesium }\end{array}$ & $\begin{array}{l}\text { Data collection: 2-D models, } \\
\text { measuring utensils and food } \\
\text { record guide used; training by } \\
\text { an RD and written instructions } \\
\text { given; participants telephoned } \\
\text { and written to during recording } \\
\text { periods; food record checked } \\
\text { by RD for accuracy and } \\
\text { completeness; weekdays and } \\
\text { weekend days recorded; } \\
\text { nutrient intake from food } \\
\text { sources only; data collected } \\
\text { in two trimesters of pregnancy }\end{array}$ \\
\hline & & No exclusions & $\begin{array}{l}\text { Energy and } \\
\text { twenty-two } \\
\text { nutrients } \\
\text { assessed }\end{array}$ & & $\begin{array}{l}\text { No statistical } \\
\text { difference in } \\
\text { mean nutrient } \\
\text { intakes between } \\
\text { second and } \\
\text { third trimesters }\end{array}$ & $\begin{array}{l}\text { Participants: random sample; } \\
\text { low socio-economic grouping } \\
\text { suggested; no exclusion criteria }\end{array}$ \\
\hline & & & $\begin{array}{l}\text { Data collected at } \\
19-21 \text { weeks and } \\
29-31 \text { weeks } \neq\end{array}$ & & & $\begin{array}{l}\text { Data analysis: statistical comparison } \\
\text { of second and third trimesters }\end{array}$ \\
\hline $\begin{array}{l}\text { Pobocik et al. (2003) } \\
\text { Guam (a US } \\
\text { territory) }\end{array}$ & $\begin{array}{l}434 \text { pregnant } \\
\text { adolescents } \\
\text { aged } 14-20 \\
\text { years }\end{array}$ & No exclusions & $24 \mathrm{~h}$ recall & $\begin{array}{l}14 \% \text { aged } \\
14-15 \text { years, } \\
47 \% 16-17 \text { years, } \\
39 \% 18-20 \text { years; } \\
71 \% \text { Chamorro } \\
\text { (indigenous Western } \\
\text { Pacific Islanders), } \\
17 \% \text { Filipino, } 3 \% \\
\text { white; } 65 \% \\
\text { enrolled in WIC }\end{array}$ & $\begin{array}{l}\text { Nutrient intakes } \\
\text { below US DRI: } \\
\text { energy, calcium, } \\
\text { iron, folate, } \\
\text { vitamin } \mathrm{E}, \\
\text { magnesium }\end{array}$ & $\begin{array}{l}\text { Data collection: dishes and food } \\
\text { models used; use of salt and } \\
\text { high-sodium condiments } \\
\text { added at table not measured; } \\
\text { nutrient intake from food } \\
\text { sources only; research } \\
\text { assistants used 'culturally } \\
\text { appropriate (data collection) } \\
\text { methods'; no details of day of } \\
\text { week data collected; data } \\
\text { collected in three trimesters } \\
\text { of pregnancy }\end{array}$ \\
\hline
\end{tabular}


the studies that assessed folate intake $(n 6)$ found that intakes fell well below the EAR of $520 \mu \mathrm{g} / \mathrm{d}$ during pregnancy. Calcium intakes were below the AI in six of the nine studies, and Pobocik et al. (2003) found that calcium intake declined with increasing age $(P<0 \cdot 0001)$. Each of the studies that measured vitamin E (Endres et al. 1985; Dunn et al. 1994; Giddens et al. 2000; Pobocik et al. 2003) and dietary fibre (Dunn et al. 1994; Giddens et al. 2000) recorded mean intakes that were below the DRI. Mean magnesium intakes were below the EAR in four of the five studies included in the review (Skinner et al. 1992; Gutierrez, 1999; Giddens et al. 2000; Pobocik et al. 2003).

\section{Discussion}

This systematic review focused only on the dietary assessment of pregnant adolescents living in industrialised countries, as cultural and socio-economic disparities between the industrialised and non-industrialised world would limit the meaningfulness of any synthesis of findings. It is likely for example, that well-nourished women raised in affluent or economically developed societies will have different nutrient needs in pregnancy from those of women from low-income developing societies (FAO/WHO/UNU, 2004). Future systematic reviews should focus on the particular nutrient intakes of pregnant adolescents living in non-industrialised countries. It is possible that the generalisability of the findings presented in this review may be limited because seven of the nine included studies were conducted in the USA. There is no a priori reason, however, why these findings would not apply to countries with similar ethnic and socio-economic demographics. The review does highlight the urgent need for further primary studies in non-US settings.

The quality of the studies included in the review was generally poor and all were limited by sampling and/or measurement bias. However, there does appear to be some consensus amongst the studies to suggest that the nutrient intakes of pregnant adolescents were low in a number of nutrients recognised to be vital for fetal growth and development during pregnancy. Crude estimates indicate that, as a percentage of the DRI, mean intakes of energy were $67-105 \%$ of the EER, intakes of iron were 49-99\% of the EAR, calcium intakes were $57-167 \%$ of the AI, folate intakes were $47-$ $86 \%$ of the EAR, intakes of vitamin E were $53-93 \%$ of the EAR and magnesium intakes were $75-106 \%$ of the EAR (percentages dependent on the age of the adolescent).

The Institute of Medicine, Food and Nutrition Board recommends that adolescent and adult women require an additional $1424 \mathrm{~kJ} / \mathrm{d}$ in the second trimester and an extra $1892 \mathrm{~kJ} / \mathrm{d}$ in the third trimester compared with the first trimester of pregnancy (Institute of Medicine, Food \& Nutrition Board, 2005). Many of the studies included in the review reported that the energy intakes of pregnant adolescents fell below the EER. Studies of energy balance during pregnancy have found that although energy expenditure increases, energy intake does not increase proportionally, yet weight gain remains normal (Prentice et al. 1989, 1996). Therefore, it is possible that the DRI overestimates the energy needs of most women during pregnancy (King, 2000). Alternatively, this incongruence could be explained by under-reporting of food intake by participants in dietary studies. It has been 
Table 3. Comparison of studies that have investigated the nutrient intakes of pregnant adolescents with US dietary reference intakes (Mean values and standard deviations or standard errors of the mean)

\begin{tabular}{|c|c|c|c|c|c|c|c|c|c|c|c|c|}
\hline & \multicolumn{4}{|c|}{ Endres et al. (1985) } & & & & & & & & \\
\hline & \multicolumn{2}{|c|}{ WIC } & \multicolumn{2}{|c|}{ Pre-WIC } & \multicolumn{2}{|c|}{ Carruth \& Skinner (1991) } & \multicolumn{2}{|c|}{ Loris et al. (1985) } & \multicolumn{2}{|c|}{ Skinner et al. (1992) } & \multicolumn{2}{|c|}{ Dunn et al. (1994) } \\
\hline & Mean & SD & Mean & SD & Mean & SD & Mean & SD & Mean & SD & Mean & SD \\
\hline Energy (kJ) & 7988 & 3136 & 8068 & 3747 & 11815 & 4333 & 9416 & 2395 & 10480 & 3408 & 11572 & 3827 \\
\hline Total protein (g) & 80.3 & 32.8 & 76.7 & 35.0 & 110 & 48 & 88 & 25 & 90 & 30 & 109.5 & 43 \\
\hline Total fat (g) & & & & & & & & & & & 118 & 56 \\
\hline Cholesterol (mg) & & & & & & & & & & & $469 \cdot 7$ & 258 \\
\hline Total carbohydrate (g) & & & & & & & & & & & 324.5 & 95 \\
\hline Dietary fibre (g) & & & & & & & & & & & $13 \cdot 3$ & 6 \\
\hline Vitamin A ( $\mu$ g RE) & $1169^{*}$ & 1313 & $1169^{*}$ & 1904 & $1440^{*}$ & 1254 & $1258^{*}$ & 989 & $1038^{*}$ & 589 & & \\
\hline Vitamin C (mg) & 85.5 & 94.1 & 99.0 & 86.8 & 133 & 104 & 121 & 78 & 104 & 80 & 111 & 95 \\
\hline Vitamin E (mg $\alpha$-tocopherol) & $8.6 \dagger$ & 13.9 & $6.4 \dagger$ & 9.8 & & & & & & & 11 & 8 \\
\hline Vitamin D $(\mu \mathrm{g})$ & $5 \cdot 2 \ddagger$ & 4.7 & $5.2 \ddagger$ & $6 \cdot 2$ & & & & & $8 \cdot 1 \neq$ & 5.5 & & \\
\hline Folate $(\mu \mathrm{g})$ & $243 \cdot 1$ & 324.5 & $242 \cdot 7$ & 240.8 & & & & & 275 & 150 & 248 & 112 \\
\hline Calcium (mg) & $918 \cdot 7$ & 572.6 & $943 \cdot 3$ & 644.9 & 1670 & 961 & 1263 & 478 & 1317 & 669 & 1188 & 762 \\
\hline Iron (mg) & 16.0 & 12.7 & $15 \cdot 1$ & 13.6 & $16 \cdot 6$ & 9.5 & 13.6 & 4 & 14.7 & $6 \cdot 1$ & 16 & 8 \\
\hline Zinc (mg) & & & & & & & & & $11 \cdot 2$ & 4.8 & 14 & 6 \\
\hline Copper (mg) & & & & & & & & & & & 1.3 & 0.7 \\
\hline Selenium $(\mu \mathrm{g})$ & & & & & & & & & & & 133 & 61 \\
\hline Thiamin (mg) & 1.6 & 1.3 & 1.5 & 1.1 & 1.86 & $1 \cdot 11$ & 1.63 & 0.50 & 1.9 & 0.7 & $2 \cdot 2$ & $1 \cdot 1$ \\
\hline Riboflavin (mg) & 2.4 & 1.6 & $2 \cdot 3$ & $2 \cdot 0$ & 3.20 & 2.05 & 2.46 & 0.76 & 2.6 & 1.1 & 2.5 & $1 \cdot 1$ \\
\hline Vitamin $\mathrm{B}_{6}(\mathrm{mg})$ & $1 \cdot 1$ & 1.3 & 1.0 & $1 \cdot 2$ & & & & & $1 \cdot 7$ & 0.8 & 2.0 & 0.9 \\
\hline Vitamin $B_{12}(\mu \mathrm{g})$ & & & & & & & & & & & $5 \cdot 1$ & $2 \cdot 2$ \\
\hline Phosphorus (mg) & 1333 & 612 & 1311 & 644 & 2049 & 906 & 1621 & 512 & & & 1744 & 815 \\
\hline Magnesium (mg) & & & & & & & & & 264 & 106 & 307 & 165 \\
\hline Potassium (mg) & & & & & 4050 & 1953 & & & & & 3484 & 1957 \\
\hline Sodium (mg) & & & & & & & & & & & 3896 & 1643 \\
\hline Niacin (mg NE) & 18.5 & 15.4 & $19 \cdot 3$ & 14.4 & 21.5 & 10.8 & 19.4 & 6.5 & $22 \cdot 0$ & 7.5 & 28 & 9 \\
\hline Pantothenic acid (mg) & & & & & & & & & & & 6 & 3 \\
\hline
\end{tabular}


Gutierrez (1999)

\begin{tabular}{|c|c|c|c|c|c|c|c|c|c|c|c|}
\hline & \multicolumn{2}{|c|}{ Job \& Capra (1995) } & \multicolumn{2}{|c|}{ 18-22 weeks gestation } & \multicolumn{2}{|c|}{$30-34$ weeks gestation } & \multicolumn{2}{|c|}{ Giddens et al. (2000) } & \multicolumn{2}{|c|}{ Pobocik et al. (2003) } & \multirow{2}{*}{$\begin{array}{l}\text { US DRI§ for pregnant } \\
\text { adolescents }\end{array}$} \\
\hline & Mean & SEM & Mean & SD & Mean & SD & Mean & SD & Mean & SD & \\
\hline Energy $(\mathrm{kJ})$ & 8930 & 398 & 10006 & 3475 & 10969 & 3341 & 9805 & 2550 & 10413 & 4425 & $\begin{array}{l}\text { First trimester } \\
\quad 9914-10061 \\
\text { Second trimester }\end{array}$ \\
\hline
\end{tabular}

Second trimester

$11338-11484$

Third trimester 11

\begin{tabular}{|c|c|c|c|c|c|c|c|c|c|c|c|}
\hline Total protein (g) & 73 & 3.4 & $111 \cdot 2$ & 45.1 & $117 \cdot 7$ & $42 \cdot 7$ & $\begin{array}{l}82 \\
97\end{array}$ & $\begin{array}{l}20 \\
28\end{array}$ & 99.0 & 44.7 & $\begin{array}{l}807-11953 \rrbracket \\
46 / 71^{\star \star}\end{array}$ \\
\hline $\begin{array}{l}\text { Total fat }(\mathrm{g}) \\
\text { Cholesterol }(\mathrm{mg})\end{array}$ & & & 324.5 & 202.9 & 413.4 & 249.9 & $\begin{array}{r}97 \\
299\end{array}$ & $\begin{array}{l}28 \\
89\end{array}$ & & & $\begin{array}{l}\text { Not determined } \\
\text { Not determined }\end{array}$ \\
\hline Total carbohydrate (g) & & & & & & & 291 & 82 & & & 135 \\
\hline Dietary fibre $(\mathrm{g})$ & & & & & & & 14 & 5 & & & $28+\dagger$ \\
\hline Vitamin A ( $\mu$ g RE) & 973 & 87 & 2492 & 3550 & 1978 & 1888 & 1053 & 572 & 1093 & 1579 & $530-550 \|$ \\
\hline Vitamin C (mg) & 135 & 22 & 252.0 & 152.4 & $230 \cdot 7$ & $140 \cdot 1$ & 128 & 62 & 167 & 183 & $66-70 \|$ \\
\hline Vitamin E (mg $\alpha$-tocopherol) & & & 10.72 & 11.39 & $11 \cdot 15$ & 10.38 & $9 \cdot 0$ & 3.5 & 8 & 8 & 12 \\
\hline Vitamin $D(\mu \mathrm{g})$ & & & & & & & $6 \cdot 4$ & $2 \cdot 6$ & & & $5.0+t$ \\
\hline Folate $(\mu \mathrm{g})$ & & & 447.4 & 319.9 & 392.5 & $181 \cdot 1$ & 312 & 138 & 292 & 242 & 520 \\
\hline Calcium (mg) & 923 & 85 & 1561 & 187 & 1655 & 800 & 989 & 332 & 743 & 575 & $1300-1000 \| \dagger \dagger$ \\
\hline Iron (mg) & $11 \cdot 2$ & 0.5 & 17.69 & 8.20 & 22.72 & 19.62 & $16 \cdot 0$ & 5.7 & 20 & 11 & $23-22 \|$ \\
\hline Zinc (mg) & 9.5 & 0.5 & 14.51 & 6.53 & $15 \cdot 29$ & 6.51 & 11.6 & $4 \cdot 2$ & 13 & 8 & $10-9.5 \|$ \\
\hline Copper (mg) & & & & & & & $1 \cdot 2$ & 0.4 & & & $0.785-0.8 \|$ \\
\hline Selenium $(\mu \mathrm{g})$ & & & & & & & 116 & 29 & & & 49 \\
\hline Thiamin $(\mathrm{mg})$ & & & & & & & $2 \cdot 1$ & 0.6 & 2.4 & $1 \cdot 2$ & $1 \cdot 2$ \\
\hline Riboflavin (mg) & & & & & & & $2 \cdot 3$ & 0.7 & $2 \cdot 1$ & 1.2 & 1.2 \\
\hline Vitamin $B_{6}(\mathrm{mg})$ & & & & & & & 1.9 & 0.6 & $2 \cdot 0$ & 1.2 & $1 \cdot 6$ \\
\hline Vitamin $B_{12}(\mu \mathrm{g})$ & & & & & & & 5.3 & 2.8 & 5.5 & 7.6 & $2 \cdot 2$ \\
\hline Phosphorus (mg) & & & & & & & 1340 & 359 & 1338 & 632 & $1055-580 \|$ \\
\hline Magnesium (mg) & & & & & & & 252 & 72 & 270 & 131 & $335-290 \|$ \\
\hline Potassium (mg) & & & & & & & & & & & $4700+\dagger$ \\
\hline Sodium (mg) & & & & & & & & & & & $1500+\dagger$ \\
\hline Niacin (mg NE) & & & & & & & $24 \cdot 1$ & 6.8 & 30 & 14 & 14 \\
\hline Pantothenic acid (mg) & & & & & & & & & & & $6+t$ \\
\hline
\end{tabular}

WIC, special supplementary nutrition program for women, infants and children; RE, retinol equivalents; NE, niacin equivalents.

${ }^{*}$ Converted to $\mu \mathrm{g}$ retinol equivalents (IU/5 $=\mu \mathrm{g}$ RE) for comparison of dietary data.

† Converted to $\mathrm{mg} \alpha$-tocopherol (I IU $=0.67 \mathrm{mg} \alpha$-tocopherol) for comparison of dietary data.

$\ddagger$ Converted to $\mu \mathrm{g}(\mathrm{I} \mathrm{IU}=0.025 \mu \mathrm{g}$ vitamin $\mathrm{D})$ for comparison of dietary data

§US dietary reference intakes (DRI) for pregnant adolescents. Unless stated otherwise, the estimated average requirements (EARs) are given

१ EER, estimated energy requirement in first/second/third trimester.

$\|$ Range denotes levels for pregnant adolescents aged $14-18$ years and 19 years.

${ }^{\star *} \mathrm{RDA}$ in first/second half of pregnancy. 
observed that some participants provide diet records that are biased to the underestimation of their true habitual intake, despite appearing highly motivated (Goldberg et al. 1993, Smithers et al. 2000, Black \& Cole, 2001). Such findings provide evidence of the need for very cautious interpretation of food-intake data. Goldberg et al. (1993) suggested that measures of energy expenditure were a more credible measure of the daily energy budget throughout gestation. In their longitudinal study of twelve women before and during pregnancy, Goldberg et al. (1993) found that there was a substantial interindividual variation in response to pregnancy, highlighting further difficulties of making prescriptive recommendations for individuals as there is no way of predicting how they will respond (metabolically and behaviourally) to pregnancy.

Iron is needed for the rapid expansion of maternal blood volume and the deposition of iron in fetal tissues. Irondeficiency anaemia is the most common nutrient deficiency in pregnancy and has also been reported to be at its peak incidence between the ages of 15 and 19 years in non-pregnant girls (Wahl, 1999), related in part to the rapid growth associated with adolescence (Lifshitz et al. 1993). The pregnant adolescent is at particular risk of developing iron-deficiency anaemia. Indeed, previous research has reported a high prevalence of anaemia (Schneck et al. 1990; Gadowsky et al. 1995) and depleted iron stores (Gadowsky et al. 1995) in pregnant adolescents. Low iron status in the pregnant adolescent has been associated with reduced fetal oxygenation (Reifsnider $\&$ Gill, 2000) and poor birth outcomes, such as a greater risk of LBW, prematurity and an increased risk of stillbirth (Ward, 2000; Tomashek et al. 2006).

During growth calcium intake is an important determinant of bone mineralisation and thus bone density. In a still growing adolescent calcium intake during pregnancy may be limited by poor maternal diet and by the need to retain enough calcium to mineralise two skeletons. Magnesium is also vital to bone quality by controlling hydroxyapatite crystal growth to prevent formation of brittle bone. However, our knowledge base to determine magnesium as opposed to calcium requirements is very weak and, without reliable magnesium status indicators, it is difficult to determine whether current intakes are adequate for optimal bone health (Weaver, 2000). Calcium also plays an important role in influencing the contractility of smooth muscle, both in the vasculature and in the uterus. It has been shown that calcium supplementation during pregnancy is associated with a reduced risk of gestational hypertension, preterm delivery and possibly pre-eclampsia (Repke \& Villar, 1991), although the beneficial effects on hypertension and preterm delivery may be confined to high-risk populations or those who have a high demand for calcium (of which pregnant adolescents are an example) (Lenders et al. 2000).

According to US recommendations, pregnant adolescents aged 14-18 years should consume $1300 \mathrm{mg}$ of calcium/d throughout pregnancy (Institute of Medicine, Food \& Nutrition Board, 1997). Those aged 19 years and above should consume $1000 \mathrm{mg}$ calcium/d. The majority of studies in the review reported that pregnant adolescents' consumption of calcium was below the recommended amount, and that intake significantly declined with increasing age (Pobocik et al. 2003). It is possible that this suboptimal intake could be due, in part, to the preferential consumption of low-calcium beverages such as soft drinks that serve to displace calcium-rich milk drinks (Harnack et al. 1999), a phenomenon which has been shown to be generally more prevalent among adolescents of lower parental occupational status compared with those with higher family affluence (Vereecken et al. 2005). The decline in calcium intake over the course of adolescence has been reported in previous studies (Gregory et al. 2000) and may be related to a decline in milk intake, which has also been observed to decline during adolescence (Harnack et al. 1999; Bowman, 2002; Striegel-Moore et al. 2006). This could reflect a decrease in breakfast consumption (Bowman, 2002; Lee \& Reicks, 2003) or increased intake of meals away from home (Bowman et al. 2004) in this population. Clearly, the low calcium intake that was observed in many adolescents could have a serious impact on the health and development of the pregnant adolescent and her fetus. Indeed, recent research has suggested that the consumption of fewer than two servings of dairy products per day by pregnant adolescents could negatively affect fetal bone development by limiting the amount of calcium provided to the fetus (Chang et al. 2003).

The consensus amongst the studies was that adolescents' folate intake did not meet the increased requirements of pregnancy. Insufficient intake of folate in the periconceptional period is linked to increased risk of neural tube defects in infants. Evidence from randomised controlled trials that supplementing with folic acid reduces the risk of neural tube defects (MRC Vitamin Study Research Group, 1991) has prompted governments around the world to advise women who could become pregnant to take folic acid supplements $(400 \mu \mathrm{g} / \mathrm{d})$, in addition to ensuring their diet is rich in foods providing folates and folic acid. However, whilst it has been demonstrated that folic acid supplementation is highly effective in optimising folate status in both adults (McNulty et al. 2000) and adolescents (Gadowsky et al. 1995), nationwide campaigns have been shown to have had limited impact in the primary target group, namely women and adolescents around the time of conception and in the early weeks of pregnancy (Buttriss, 2004). Surveys conducted in the UK, Australia, the USA and Puerto Rico indicate that only around $30 \%$ of the pregnant population use folic acid correctly, i.e. periconceptionally (Wild et al. 1997; Miller et al. 2000; Honein et al. 2001; De La Vega et al. 2002). This figure could be even lower in the pregnant adolescent population, with only $14 \%$ of 16-19 year olds reporting being aware of the need to increase folate intake before conception (Wild et al. 1996).

The vitamin $\mathrm{E}$ intakes of the pregnant adolescents were below the recommended intake amounts. These estimates of intake may be artificially low, however, as the dietary supply of vitamin E is difficult to assess. Food composition data are poor and reported intake of fat and oil, the major sources of vitamin $\mathrm{E}$ in the diet, may be underestimated (Gutierrez \& King, 1993). Biochemical or clinical evidence of vitamin E deficiency in industrialised countries is rare.

There was limited evidence to suggest that adolescents' nutrient intakes varied according to the stage of pregnancy. Job \& Capra (1995), Gutierrez (1999) and Giddens et al. (2000) failed to find a significant difference in intakes between trimesters. However, Pobocik and colleagues' (2003) study of 434 adolescents reported that iron intakes were lower in the second compared with the third trimester $(P<0.05)$. Differences also existed for thiamin, riboflavin and phosphorus, but no further 
details were provided as intakes of these nutrients were above the recommended intake amounts. It is possible that the absence of significant differences observed in the majority of the studies was due to their small sample size, and further large-scale studies are necessary in order to elucidate this.

There were a number of methodological limitations in the studies that should be considered when interpreting these results. The application of exclusion criteria and control of potential confounding variables was limited in most studies, thereby limiting the reliability of the data. All of the studies were subject to sampling bias, with most utilising convenience sampling methods. Only one study used random sampling (Giddens et al. 2000). This study, however, was part of a larger randomised controlled trial on calcium intake where participants were prescribed multivitamin/mineral supplements and, consequently, reported a high use of nutrient supplementation (79\% reported a high compliance in taking the supplement). This is in contrast to an earlier study that reported limited use of supplements by pregnant adolescents (Skinner \& Carruth, 1991). It is likely that this relatively highly motivated sample of adolescents had dietary habits that differ from those of pregnant adolescents who do not take supplements. Previous research has confirmed that young people who consume dietary supplements tend to have higher intakes of vitamins from food sources than nonsupplement users (Gregory et al. 2000). Unfortunately, Giddens et al. did not examine the dietary differences between supplement users and non-users, and therefore potential differences in nutrient intake cannot be ascertained.

The participants in the included studies tended to be older adolescents, with a mean age of between 16 and 17 years. It is likely that the nutritional requirements of a younger adolescent group (which are needed to sustain their own growth, as well as the growth of their fetus) are different from those of an older adolescent group. Only two studies conducted comparisons of the nutrient intakes of older $v$. younger pregnant adolescents. Job \& Capra (1995) compared two groups of adolescents; one aged 15-16 years ( $n$ 23) and another aged 17-18 years ( $n$ 47), and found no significant differences between age groups, possibly due to the relatively small sample sizes of the groups. In a larger study, Pobocik et al. (2003) found two differences when comparing the nutrient intakes of adolescents aged 14-15 years ( $n$ 61), 16-17 years ( $n$ 203) and 18-20 years ( $n$ 170). They found that calcium intake significantly declined with increasing age, and phosphorus 'varied' with age (but with no further detail provided). Again, due to the lack of available data, conclusions cannot be drawn and further large-scale studies are necessary.

Socio-economic factors are known to exert a strong influence on nutrient intake. Low-income groups are less likely to eat sufficient quantities of fruit and vegetables (Henderson et al. 2003) and are more likely to consume whole milk, table sugar and sugar confectionary than higher income groups (Gregory et al. 2000). Barriers to good nutritional practices of women living in conditions of material deprivation include problems with access, cost and storage of food (Reid \& Adamson, 1997). It has been reported that a high proportion of low-income pregnant adolescents miss meals and resort to buying less healthy 'cheap filler' foods when money runs out (Burchett \& Seely, 2003). Over time, such ways of 'managing' poverty can become second nature, despite the potential costs to the adolescents' physical and emotional well-being (Attree, 2005). In addition, whilst peer influences may become more dominant as adolescents get older (Buttriss, 2002), the influence of the family on their food choices and eating behaviours should not be overlooked. A recent study has shown, for example, that parental presence at the evening meal has been positively associated with adolescents' higher consumption of fruit, vegetables and dairy foods (Videon \& Manning, 2003). Few of the studies in this review provided sufficient amount of detail regarding the socio-economic and living circumstances of their participants. Without this information, it is not possible to identify accurately adolescents who are in need of specific nutritional intervention.

The majority of studies used data collection methods that were subject to measurement bias. Five studies used $24 \mathrm{~h}$ recall to collect dietary data, and three of those took measurements on a single day only. It is widely acknowledged that the $24 \mathrm{~h}$ recall method of nutrient intake assessment suffers from many limitations. The success of this method depends on the degree of motivation of the respondent, the persistence of the interviewer, and the participants' memory and their ability to estimate portion sizes. The $24 \mathrm{~h}$ recall is also subject to errors brought about by alterations in the food habits of participants. In addition, the $24 \mathrm{~h}$ recall method is not indicative of an individual's overall diet, as recording may not take place on a 'typical' day and therefore substantial error may be introduced when assessing a single day's diet (Nelson \& Bingham, 1997). The size of the group required for a single $24 \mathrm{~h}$ recall to determine the mean usual nutrient intake of a group depends on the day-to-day variation in food intake between participants (i.e. between-subject variation), which is, in turn, affected by the nutrient under study. Generally, for example, for nutrients found in high concentrations in only a few foods, such as vitamin A, vitamin D and cholesterol, the number of records needed is much greater than for those found in a wide range of foods (e.g. protein) (Gibson, 2005). Indeed, large standard deviations can be observed in the included studies for many of the vitamins and minerals, indicating the presence of considerable between-subject variation as well as being reflective of those nutrients found in high amounts in relatively few foods. In addition, many studies failed to take account of weekend and seasonal variations in nutrient intake. Group mean nutrient intakes can vary according to the day of the week and the season in which they were consumed, although this effect is not exhibited by all nutrients (Gibson et al. 1985).

Thus, it is clear that, at present, there is a paucity of highquality research that uses adequate methodological techniques to provide us with a clear description of pregnant adolescents' nutrient intake. Further well-designed and controlled studies are needed which take into account the influence of adolescent age, the stage of pregnancy, and weekly and seasonal variations in nutrient intake. In order to assess the effect of pregnant adolescent's nutrient intakes and the interactions of different nutrients on nutritional status, studies should also include biochemical indices of nutritional status and followup studies on maternal and neonatal outcomes. The various socio-cultural influences affecting the pregnant adolescent's nutrient intake should also be investigated further.

The nutritional challenges that face the pregnant adolescent are unique. The increased nutrient demands of pregnancy, 
together with the increased nutrient demands of the still-growing adolescent, may generate competition for nutrients between mother and fetus. Although relatively limited, the current research seems to suggest that pregnant adolescents' diets are suboptimal, particularly with regards to energy, iron, folate, calcium, vitamin $\mathrm{E}$ and magnesium. As up to $75 \%$ of adolescents do not plan pregnancy (Goldberg, 2002), the issue of periconceptional nutrition is problematic. A UK survey of 674 adolescents (aged 14-15 years) on their perceptions of what constitutes a healthy pregnancy revealed that $70 \%$ of respondents thought that the optimum time to initiate changes in what a woman eats and drinks to ensure a healthy pregnancy was when pregnancy had been confirmed (Edwards et al. 1997). This suggests that the benefits of pre-conceptual nutrition are not well understood by this population. A further complication is that many adolescents may be unaware of their pregnancy or may not have accessed services in their first trimester, so providing the appropriate support to these individuals may be difficult.

Nutrition in adolescent pregnancy must be viewed within a biopsychosocial context, since it has consistently been shown that there are multiple influencing factors that play a role in the eating behaviour and subsequent nutritional status of the pregnant adolescent. Eating behaviours are likely to be related to other, often 'risky', behaviours displayed in adolescents and should not be viewed in isolation (Irwin et al. 1997). Achieving dietary change in this particularly vulnerable section of the population, many of who are from disadvantaged backgrounds (Social Exclusion Unit, 1999), presents a major public health challenge. Biopsychosocial factors often experienced by such groups, including low levels of disposable income, unemployment, poor housing, suboptimal mental and physical health and limited access to a wide variety of reasonably priced foods, all contribute to difficulties in tackling behavioural change (Symon \& Wrieden, 2003). These factors, in turn, lead to increasing health inequalities (Acheson, 1998). An important factor that should be considered when developing appropriate and effective strategies to promote healthy eating in pregnant adolescents is the heterogeneity of the group. Factors affecting food choices vary considerably, depending on the individual's particular circumstances. Family and peers are likely to have a strong influence on the eating habits of most pregnant adolescents. Poverty is a significant factor that limits the ability of some pregnant adolescents to eat a healthy diet, even in those who aspire to it.

There are numerous barriers to optimal nutrition in adolescent pregnancy, and any intervention should consider these carefully. Overcoming the barriers in order to achieve improved nutrition in pregnancy among adolescents requires multidisciplinary collaborations of adolescent health care providers, academics, professional organisations, policy makers, industry and service users. Certainly, more needs to be done at a policy level, both with regards to enabling adolescents' access to optimal nutrition and in modifying the nutrition message that adolescents receive. Governments around the world should ensure that there is consistency of food and nutrition messages in schools, for example, to include the curriculum, food provision in the canteen, vending machine policies, breakfast clubs, snacking and lunchbox policies. Any intervention that aims to encourage changes in lifestyle should be multifactorial and incorporate measures to improve the socio-economic circumstances of adolescents and their families. Only once this is achieved can adolescent nutrition, and adolescent nutrition in pregnancy, be significantly and sustainably optimised.

\section{References}

Acheson D [chairman] (1998) Independent Inquiry into Inequalities in Health. London: H.M. Stationery Office.

Attree P (2005) Low-income mothers, nutrition and health: a systematic review of qualitative evidence. Maternal and Child Nutrition 1, 227-240.

Black AE \& Cole TJ (2001) Biased over- or under-reporting is characteristic of individuals whether over time or by different assessment methods. $J$ Am Diet Assoc 101, 70-80.

Bowman SA (2002) Beverage choices of young females: changes and impact on nutrient intakes. J Am Diet Assoc 102, 1234-1239.

Bowman SA, Gortmaker SL, Ebbeling CB, Pereira MA \& Ludwig DS (2004) Effects of fast-food consumption on energy intake and diet quality among children in a national household survey. Pediatrics 113, 112-118.

Burchett H \& Seeley A (2003) Good Enough to Eat? The Diet of Pregnant Teenagers. London: Maternity Alliance/Food Commission.

Buttris J (2002) Nutrition, health and schoolchildren. Nutr Bull 27, 275-305.

Buttriss J (2004) Strategies to increase folate/folic acid intake in women: an overview. Nutr Bull 29, 234-244.

Carruth BR (1981) Smoking and pregnancy outcome of adolescents. J Adolesc Health Care 2, 115-120.

Carruth BR \& Skinner JD (1991) Pregnant adolescents report infrequent use of sugar substitutes. J Am Diet Assoc 91, 608-610.

Casanueva E, Magana L, Pfeffer F \& Baez A (1991) Incidence of premature rupture of membranes in pregnant women with low leukocyte levels of vitamin C. Eur J Clin Nutr 45, 401-405.

CASP (2004) 12 questions to help you make sense of a cohort study. Available at: http://www.phru.nhs.uk/casp/critical_appraisal_tools. htm\#cohort. Learning \& Development Public Health Resource Unit, Oxford.

Cavadini C, Siega-Riz AM \& Popkin BM (2000) US adolescent food intake trends from 1965 to 1996. Arch Dis Child 83, 18-24.

Chang S-C, O'Brien KO, Nathanson MS, Caulfield LE, Mancini J \& Witter FR (2003) Fetal femur length is influenced by maternal dairy intake in pregnant African American adolescents. Am J Clin Nutr 77, 1248-1254.

De La Vega A, Salicrup E \& Verdiales M (2002) A nationwide program for the use of preconceptional folic acid to prevent the development of open neural tube defects. Who is really using folic acid? $P R$ Health Sci J 21, 7-9.

Dunn C, Kolasa K, Dunn PC \& Ogle MB (1994) Dietary intake of pregnant adolescents in a rural southern community. $J$ Am Diet Assoc 94, 1040-1041.

Edwards G, Stainisstreet M \& Boyes E (1997) Adolescents' ideas about the health of the fetus. Midwifery 13, 17-23.

Endres J, Dunning S, Poon S, Welch P \& Duncan H (1987) Older pregnant women and adolescents: nutrition data after enrollment in WIC. J Am Diet Assoc 87, 1011-1019.

Endres JM, Poell-Odenwald K, Sawicki M \& Welch P (1985) Dietary assessment of pregnant adolescents participating in a supplementalfood program. J Reprod Med 30, 10-17.

FAO/WHO/UNU (2004) Human Energy Requirements: Report of a Joint FAO/WHO/UNU Expert Consultation. Food and Nutrition Technical Report Series 1. http://www.fao.org/docrep/007/ y5686e/y5686e00.htm [accessed 21 August 2006].

Frisancho AR, Matos J \& Flegel P (1983) Maternal nutritional status and adolescent pregnancy outcome. Am J Clin Nutr 38, 739-746.

Gadowsky SL, Gale K, Wolfe SA, Jory J, Gibson R \& O'Connor DL (1995) Biochemical folate, B12 and iron status of a group of 
pregnant adolescents accessed through the public health system in Southern Ontario. J Adolesc Health 16, 465-474.

Gibson RS, Gibson IL \& Kitching J (1985) A study of inter- and intrasubject variability in seven-day weighed dietary intakes with particular emphasis on trace elements. Biol Trace Elem Res 8, 79-91.

Gibson RS (2005) Principles of Nutritional Assessment, 2nd ed., Oxford: Oxford University Press.

Giddens JB, Krug SK, Tsang RC, Guo S, Miodovnik M \& Prada JA (2000) Pregnant adolescent and adult women have similarly low intakes of selected nutrients. J Am Diet Assoc 100, 1334-1340.

Goldberg G (2002) Nutrition in pregnancy and lactation. In Nutrition Through the Life Cycle, pp. 63-90 [P Shetty, editor]. Leatherhead, UK: Leatherhead Publishing.

Goldberg G, Prentice AM, Coward WA, Davies HL, Murgatroyd PR, Wensing C, Black AE, Harding M \& Sawyer M (1993) Longitudinal assessment of energy expenditure in pregnancy by the doubly labelled water method. Am J Clin Nutr 57, 494-505.

Gregory J, Lowe S, Bates C, Prentice A, Jackson L, Smithers G, Wenlock R \& Farron M (2000) Report of the Diet and Nutrition Survey Volume 1, National Diet and Nutrition Survey: Young People Aged 4 to 18 Years. London: The Stationery Office.

Gutierrez YM (1999) Cultural factors affecting diet and pregnancy outcome of Mexican American adolescents. J Adolesc Health 25, 227-237.

Gutierrez Y \& King JC (1993) Nutrition during adolescent pregnancy. Pediatr Ann 22, 99-108.

Hamlyn B, Brooker S, Oleinikova K \& Wands S (2002) Infant Feeding 2000. London: The Stationery Office.

Harnack L, Stang J \& Story M (1999) Soft drink consumption among US children and adolescents: nutritional consequences. $J$ Am Diet Assoc 99, 436-441.

Hediger ML, Scholl TO, Ances IG, Belsky DH \& Salmon RW (1990) Rate and amount of weight gain during adolescent pregnancy: associations with maternal weight-for-height and birth weight. Am J Clin Nutr 52, 700-793.

Henderson J, Irving K, Gregory J, Bates CJ, Prentice A, Perks J, Swan G \& Farron M (2003) The National Diet and Nutrition Survey: Adults Aged 19-64 Years, Volume 3: Vitamin and Mineral Intakes and Urinary Analyte. London: The Stationery Office.

Honein MA, Paulozzi LJ, Matthews TJ, Erickson JD \& Wong LY (2001) Impact of folic acid fortification of the US food supply on the incidence of neural tube defects. J Am Med Assoc $\mathbf{2 8 5}$, 2981-2986.

Institute of Medicine, Food and Nutrition Board (1997) Dietary Reference Intakes for Calcium, Phosphorus, Magnesium, Vitamin $D$, and Fluoride. Washington, DC: National Academy Press.

Institute of Medicine, Food and Nutrition Board (1998) Dietary Reference Intakes for Thiamin, Riboflavin, Niacin, Vitamin B6, Folate, Vitamin B12, Pantothenic Acid, Biotin, and Choline. Washington, DC: National Academy Press.

Institute of Medicine, Food and Nutrition Board (2000) Dietary Reference Intakes for Vitamin C, Vitamin E, Selenium, and Carotenoids. Washington, DC: National Academy Press.

Institute of Medicine, Food and Nutrition Board (2000b) Dietary Reference Intakes for Vitamin A, Vitamin K, Arsenic, Boron, Chromium, Copper, Iodine, Iron, Manganese, Molybdenum, Nickel, Silicon, Vanadium, and Zinc. Washington, DC: National Academy Press.

Institute of Medicine, Food and Nutrition Board (2005a) Dietary Reference Intakes for Energy, Carbohydrate, Fiber, Fat, Fatty Acids, Cholesterol, Protein, and Amino Acids (Macronutrients). Washington, DC: National Academy Press.

Irwin CE, Igra V, Eyre S \& Millstein S (1997) Risk-taking behavior in adolescents: the paradigm. Ann NY Acad Sci 817, 1-35.

Job J \& Capra S (1995) Nutritional assessment of pregnant teenagers attending a metropolitan, public, maternity hospital in Brisbane 1. Nutrient intakes. Aust J Nutr Diet 52, 76-82.
Kelly A, Kevany J, de Onis M \& Shah PM (1996) A WHO collaborative study of maternal anthropometry and pregnancy outcomes. Int J Gynecol Obst 53, 219-233.

King JC (2000) Physiology of pregnancy and nutrient metabolism. Am J Clin Nutr 71, 1218S-12125S.

King JC (2003) The risk of maternal nutritional depletion and poor outcomes increases in early or closely spaced pregnancies. $J$ Nutr 133, 1732S-1736S.

Lee S \& Reicks M (2003) Environmental and behavioural factors are associated with the calcium intake of low-income adolescent girls. $J$ Am Diet Assoc 103, 1526-1529.

Lenders CM, Hediger ML, Scholl TO, Khoo C-S, Slap GB \& Stallings VA (1994) Effect of high-sugar intake by low-income pregnant adolescents on infant birth weight. $J$ Adolesc Health 15, 596-602.

Lenders CM, Hediger ML, Scholl TO, Khoo C-S, Slap GB \& Stallings VA (1997) Gestational age and infant size at birth are associated with dietary sugar intake among pregnant adolescents. J Nutr 127, 1113-1117.

Lenders CM, McElrath TF \& Scholl TO (2000) Nutrition in adolescent pregnancy. Curr Opin Pediatr 12, 291-296.

Lifshitz F, Tarim O \& Smith MM (1993) Nutrition in adolescence. Adolesc Endocrinol 22, 673-683.

Loris P, Dewey KG \& Poirier-Brode K (1985) Weight gain and dietary intake of pregnant teenagers. $J$ Am Diet Assoc 85, 1296-1305.

Lytle LA (2002) Nutritional issues for adolescents. J Am Diet Assoc 102, S8-S12.

McNulty H, Cuskelly GJ \& Ward M (2000) Response of red blood cell folate to intervention: implications for folate recommendations for the prevention of neural tube defects. Am J Clin Nutr 71, 1308S-1311S.

Miller M, Bower C \& Payne J (2000) Folate-related knowledge and practices of Western Australian women before and during pregnancy. Teratology 62, 367.

MRC Vitamin Study Research Group (1991) Prevention of neural tube defects: results of the Medical Research Council Vitamin Study. Lancet 338, 131-137.

Naeye RL (1981) Teenage and pre-teenaged pregnancies: consequences of the fetal-maternal competition for nutrients. Pediatrics 67, 146-159.

Nelson M \& Bingham SA (1997) Assessment of food consumption and nutrient intake. In Design Concepts in Nutritional Epidemiology, 2nd ed., pp. 123-169 [BM Margetts and M Nelson, editors]. Oxford: Oxford University Press.

NHS Centre for Reviews and Dissemination (2001) Undertaking Systematic Reviews of Research on Effectiveness: Guidance for Those Carrying Out or Commissioning Reviews. York, UK: NHS CRD.

Office for National Statistics (2004) Health Statistics Quarterly, Winter 2004. London: The Stationery Office.

Office for National Statistics (2005) Teenage Conception Statistics for England 1998-2003. http://www.dfes.gov.uk/teenagepregnancy/ [accessed 5 February 2006].

Olausson PO, Cnattingius S \& Haglund B (1999) Teenage pregnancies and risk of late fetal death and infant mortality. $\mathrm{Br}$ J Obstet Gynaecol 106, 116-121.

Pobocik RS, Benavente JC, Boudreau NS \& Spore CL (2003) Pregnant adolescents in Guam consume diets low in calcium and other micronutrients. $J$ Am Diet Assoc 103, 611-614.

Pope JE, Skinner JD \& Carruth BR (1997) Adolescents' self-reported motivations for dietary change during pregnancy. Journal of Nutrition Education 29, 137-144.

Prentice AM, Goldberg GR, Davies HL, Murgatroyd PR \& Scott W (1989) Energy sparing adaptations in human pregnancy assessed by whole body calorimetry. Br J Nutr 62, 5-22.

Prentice AM, Spaaij CJK, Goldberg GR, Poppitt SD, van Raaij JMA, Swann D \& Black AE (1996) Energy requirements of pregnant and lactating women. Am J Clin Nutr 50, S82-S111. 
Reid M \& Adamson H (1997) Opportunities for and Barriers to Good Nutritional Health in Women of Childbearing Age, Pregnant Women, Infants Under 1 and Children Aged 1 to 5. London: Health Education Authority.

Reifsnider E \& Gill SL (2000) Nutrition for the childbearing years. J Obstet Gynecol Neonatal Nurs 29, 43-55.

Repke JT \& Villar J (1991) Pregnancy-induced hypertension and low birth weight: the role of calcium. Am J Clin Nutr 54, 237S-241S.

Schneck ME, Sideras KS, Fox RA \& Dupuis L (1990) Low income pregnant adolescents and their infants: dietary findings and health outcomes. J Am Diet Assoc 90, 555-558.

Scholl TO, Hediger ML \& Ances IG (1990) Maternal growth during pregnancy and decreased birth weight. Am J Clin Nutr 51, 790-793.

Scholl TO, Hediger ML, Schall JI, Khoo CS \& Fischer RL (1994) Maternal growth during pregnancy and competition for nutrients. Am J Clin Nutr 60, 183-188.

Scholl TO, Stein TP \& Smith WK (2000) Leptin and maternal growth during adolescent pregnancy. Am J Clin Nutr 72, 1542-1547.

Skinner JD \& Carruth BR (1991) Dietary quality of pregnant and nonpregnant adolescents. J Am Diet Assoc 91, 718-720.

Skinner JD, Carruth BR, Pope J, Varner L \& Goldberg D (1992) Food and nutrient intake of white, pregnant adolescents. J Am Diet Assoc 912, 1127-1129.

Smithers G, Gregory JR, Bates CJ, Prentice A, Jackson LV \& Wenlock R (2000) The National Diet and Nutrition Survey: young people aged $4-18$ years. Nutr Bull 25, 105-111.

Social Exclusion Unit (1999) Teenage Pregnancy: Report by the Social Exclusion Unit. London: HMSO.

Stedman's Medical Dictionary, 2nd ed. (2004) Wilmington, MA: Houghton Mifflin Company.

Stevens-Simon C, Fullar S \& McAnarney ER (1992) Tangible differences between adolescent-oriented and adult-oriented prenatal care. J Adolesc Health 13, 298-302.

Story M \& Alton I (1995) Nutrition issues and adolescent pregnancy. Nutr Today 30, 142-151.

Striegel-Moore RH, Thompson D, Affenito SG, Franko DL, Obarzanek E, Barton BA, Schreiber GB, Daniels SR, Schmidt M \& Crawford PB (2006) Correlates of beverage intake in adolescent girls: The National Heart, Lung, and Blood Institute Growth and Health Study. J Pediatr 148, 183-187.

Symon AG \& Wrieden WL (2003) A qualitative study of pregnant teenagers' perceptions of the acceptability of a nutritional education intervention. Midwifery 19, 140-147.

Teenage Pregnancy Unit (2004) Teenage Pregnancy: An Overview of the Research Evidence. London: Health Development Agency.

Tomashek KM, Ananth CV \& Cogswell ME (2006) Risk of stillbirth in relation to maternal haemoglobin concentration during pregnancy. Maternal Child Nutr 2, 19-28.

Umans JG \& Lindheimer MD (2001) Antihypertensive therapy in pregnancy. Curr Hypertens Rep 3, 392-399.

UNICEF (2001) A League Table of Teenage Births in Rich Nations. Innocenti Report Card No 3, UNICEF Innocenti Research Centre, Florence. www.unicef-icdc.org/publications/index.html [accessed 6 July 2005].

Vereecken CA, Inchley J, Subramanian SV, Hublet A \& Maes L (2005) The relative influence of individual and contextual socioeconomic status on consumption of fruit and soft drinks among adolescents in Europe. Eur J Public Health 15, 224-232.

Videon TM \& Manning CK (2003) Influences on adolescent eating patterns: the importance of family meals. J Adolesc Health 32, 365-373.

Wahl R (1999) Nutrition in the adolescent. Pediatr Ann 28, $107-111$.

Ward B (2000) Sandwell report in note 31. In Poverty in Plenty: A Human Development Report for the UK, [J Seymour, editor]. London: Earthscan.

Weaver C (2000) Calcium and magnesium requirements of children and adolescents and peak bone mass. Nutrition 16, 514-516.

WHO (1995) Maternal anthropometry and pregnancy outcomes. A WHO Collaborative Study. WHO Bull 73, Suppl. 1 1-98.

Wild J, Schorah CJ, Maude K \& Levene MI (1996) Folate intake in young women and their knowledge of pre-conceptional folate supplementation to prevent neural tube defects. Eur J Obstet Gynecol Reprod Biol 70, 185-189.

Wild J, Sutcliffe M, Schorah CJ \& Levene MI (1997) Prevention of neural tube defects. Lancet 350, 30-31. 